In conclusion, the agreement between equations (1) and (2) in Alpan's comment is not accidental. Rather, it confirms the detailed analysis of the Paper which shows that, to a good approximation, the response of a rocking body on a non-homogeneous elastic half-space can be estimated from the static stress distribution of the same body on a homogeneous elastic half-space whose modulus is the same as the surface modulus of the non-homogeneous medium. The range of validity of the approximation and the physical interpretation of the behaviour of the system have been established in the Paper. These cannot be obtained from simple models.

\title{
Deformation and failure pattern in an anisotropic kaolinite clay
}

\author{
SANKARAN, K. S. and BHASKARAN, R. (1973). Géotechnique 23, No. 1, 113-117.
}

\section{P. K. De, Sir William Halcrow \& Partners, Aberdare, Glamorgan}

While conducting unconfined compression tests the Authors observed the deformation and failure patterns of anisotropic kaolinite clay. Unfortunately the observations were visual, and from the experience I have gained by investigating shear deformation and failure patterns of shear box samples of kaolinite clay it is suggested that any conclusions drawn from such visual observations may not always be valid and should be confirmed by an optical or electron microscopic examination. This is to say that, in general, the visual failure patterns seen on the surface of samples do not represent true failure patterns.

The main subject of the Note is anisotropy but apparently the Authors did not establish the degree of anisotropy of the kaolinite clay used in the investigation. This could be defined in many ways, such as by the ratio of the minimum and maximum intensities of transmitted polarized light or more directly by transmission or scanning electron micrographs.

The ratio of the transmitted polarized light intensities

$$
\beta=I_{\min } / I_{\max }
$$

is a measure of the degree of particle orientation in which $\beta$ approaches zero for 'perfect' orientation, and unity for 'random' arrangements. If there is no optical or electron microscopic investigation of the degree of anisotropy of the clay then the analogy of fabric with a pack of cards does not convey any message other than the assumption that the kaolinite clay particles were perfectly orientated. Surely this is an oversimplification of the actual fabric of the clay.

The Authors' invariable assumption that the failure planes were formed at, or after, the peak compression strength needs some elaboration. It would be interesting to know, for example, how the formation of shear planes was investigated and whether this was done by microscopic examination or by visual observation as shown in the Figs 1, 2 and 3 of the Paper.

\section{Author's reply to P. K. De}

It appears that De does not follow the main theme of the Note. Certain interesting deformation and failure patterns were presented, on the macro scale, in specimens extracted in different directions, when testing was carried out in a simple stress configuration such as the unconfined compression test with a minimum of end restraint. Though of added interest, no 
attempt has been made to study the reorientation of particles in these specimens, either during shear or at failure. The parallel De seeks with his own experience with shear box tests (with constraints and complex stress configurations), where he has tried to study the shear discontinuities and the microstructural patterns (Foster and De, 1971), is thus not relevant to our study.

We are satisfied that a degree of parallel orientation exists in the different specimens and have tried to explain the observed macroscopic deformations and failure patterns observed in terms of the preferred orientation of particles within the specimens.

We are also well aware of the necessity of studying microstructure to explain the macroscopic behaviour of clays and interesting results have been obtained at the Indian Institute of Technology, Madras in this connection (Sankaran and Rao, 1973a, b).

\section{REFERENCES}

Foster, R. H. \& De, P. K. (1971). Optical and electron microscopic investigation of shear induced structures in lightly consolidated (soft) and heavily consolidated (hard) kaolinite. Clay and clay minerals 19, $31-47$.

Sankaran, K. S. \& Rao, D. V. (1973a). Quantitative estimation of particle orientation of montmorillonite by optical and X-ray diffraction techniques. Research Report Publication 3/73, Soil Engineering Laboratory, Indian Institute of Technology, Madras, India.

Sankaran, K. S. \& Rao, D. V. (1973b). A microscopic model of expansive clays. To be published in Proc. Internat. Conf. expansive soils, Haifa, Israel, 1973.

\section{Some deviations from isotropy in a lightly over- consolidated clay}

MITCHELL, R. J. (1972), Géotechnique 22, No. 3, 459-467.

Discussion by Bhaskaran (1973). Géotechnique 23, No. 2, 292-294.

\section{Author's reply to R. Bhaskaran}

Bhaskaran's interest in and contributions to the subject of anisotropy, as outlined in his discussion, are most welcome. Such efforts are providing background for investigation of the more complex problems of anisotropic response of natural soils.

In his discussion, Bhaskaran questions the necessity of doing isotropic reconsolidation tests on both vertical and horizontal specimens. Apart from the practical requirement of ascertaining that the observations are not being unduly influenced by end restraint, the behaviour of an orientated particulate material under radially symmetric stress conditions may well differ from that expected of an elastic cross-anisotropic material. If the specimen retains a right circular shape the radial and circumferential strains will, from continuity, be equal. The method of analyses adopted in the Paper was chosen because measured deviations from a right circular shape were not noted. The fabric of kaolin after one-dimensional consolidation appears to be composed of packets of preferentially aligned particles dispersed in a random matrix. The observed data appear to be consistent with the possible behaviour of such a system. It appears that this problem requires further experimental evaluation by combining the results of true triaxial tests with the standard triaxial ones, and by also correlating macroscopic and microscopic observations. 